\title{
Caracterização de resíduo de ETA visando sua disposição na saída de Lagoas de Estabilização
}

\author{
Characterization of WTP residual aiming its disposal in output of stabilization ponds
}

\author{
Lorena Acelina Soares', Paulo Sergio Scalize ${ }^{2}$, Antonio João Carvalho Albuquerque ${ }^{3}$ \\ 'Mestre em Engenharia do Meio Ambiente - Universidade Federal de Goiás - Brasil \\ ${ }^{2}$ Doutor em Hidraulica e Saneamento pela USP São Carlos. Professor adjunto da Escola de Engenharia Civil da Universidade Federal de \\ Goiás. Professor do Curso de Mestrado em Engenharia do Meio Ambiente (PPGEMA). - Universidade Federal de Goiás - Brasil \\ ${ }^{3}$ Doutor em Engenharia Civil pela Universidade da Beira Interior - Professor Auxiliar e Investigador Sénior- . Universidade da Beira \\ Interior - Portugal
}

\begin{abstract}
Resumo
As ETAs são responsáveis pela produção de água potável a ser consumida pela população, o que gera benefícios sociais, porém, como em toda indústria de transformação, resíduos são gerados e há a necessidade de dispô-los adequadamente sem prejudicar o meio ambiente. Quando se trata do gerenciamento de resíduos sólidos, e aí está incluído a sua destinação, é necessário conhecer as suas características a fim de se definir pelo melhor tratamento e disposição. Com base nisso, este trabalho teve por objetivo caracterizar o resíduo da ETA, localizada no estado de Goiás, Brasil, que emprega em seu tratamento o coagulante sulfato de alumínio. Foram realizadas análises físico-químicas e estruturais, sendo possível verificar que o resíduo é constituído por argila (incluindo principalmente os minerais caulinita e haloisita), silte e areia. O resíduo foi determinado como não plástico. Por análise EDS foi detectada a presença de alumínio, que está relacionado à remoção de fósforo em água e sedimentos, o que favorece a sua reutilização no tratamento de águas residuais.
\end{abstract}

Palavras-chave: descarga de decantador, estação de tratamento de água, lama, lodo, resíduo.

\begin{abstract}
The WTP are responsible for the production of drinking water to be consumed by the population, which generates social benefits, however, as in any manufacturing industry, waste is generated and there is the need to dispose them properly without harming the environment. The solid waste management included waste destination and for this stage is necessary to know the waste characteristics in order to define the best treatment and disposal. This study aimed to characterize the WTPS produced by a WTP in the state of Goiás, Brazil, that employs in their treatment the coagulant aluminum sulfate. Physico-chemical analyses and structural properties were verified, showing that the residue consists of clay (mainly the mineral kaolinite and halloysite), silt and sand. The residue was determined as not plastic. The EDS analysis detected the presence of aluminum, which is related to the phosphorus removal in water and sediment, which facilitates their reuse in the wastewater treatment
\end{abstract}

Keywords: discharge of decanter, Water Treatment Plant, mud, sludge, residue. 


\section{INTRODUÇÃO}

Os resíduos das estações de tratamento de água (RETAs) são sub-produtos do processo de potabilidade da água, sendo considerados o maior passivo ambiental do setor, visto que a preocupação com sua correta disposição, sem prejudicar o meio ambiente, vem ganhando crescente atenção, devido a sua composição, com a presença de metais. No Brasil, o RETA é classificado como resíduo sólido pela NBR 10004 (2004), devendo ser disposto adequadamente, porém ainda é comum o lançamento em corpos hídricos, prejudicando a biota aquática.

Os problemas ambientais causados pela incorreta disposição dos RETAs podem ser físicos, químicos e biológicos. Essa disposição inadequada pode ocasionar o aumento na quantidade de sólidos em suspensão, o assoreamento do leito do rio, alterações na cor da água, o aumento das concentrações de alumínio ou ferro, que podem ser tóxicos para organismos aquáticos, o aumento da demanda de oxigênio, a inibição da atividade biológica, além disso, podem ter influência negativa em áreas de criação e desova de peixes (EPA, 2011; SILVA JUNIOR, 2003).

A preocupação em torno da sua disposição torna-se maior devido a probabilidade de aumento na geração desses resíduos, visto o aumento populacional, exigindo maior demanda de água produzida, e a tendência a padrões mais restritivos para qualidade da água potável, o que poderá requerer uma maior utilização de produtos químicos. Dessa forma, encontrar opções de reutilização benéfica irá se tornar fundamental com as pressões ambientais e econômicas limitando as opções de eliminação do resíduo material (IPPOLITO; BARBARICK; ELLIOTT, 2011).

Nesse aspecto mudanças positivas ocorreram quando se compara o RETA gerado hoje com o dos anos 90, pois tem-se percebido a redução na concentração dos metais cádmio, chumbo, cromo, entre outros, devido a melhor qualidade dos produtos químicos usados atualmente. Essas mudanças são positivas garantindo ainda mais segurança na reutilização desse material (IPPOLITO; BARBARICK; ELLIOTT, 2011).

Como alternativa de reutilização ou disposição muitos estudos tem avaliado o RETA como matéria-prima para outros processos, como na fabricação de tijolos e materiais cerâmicos, auxiliar de coagulação e floculação, na recuperação de áreas degradadas e sua disposição em estações de tratamento de esgotos (ETEs). Independente do uso e de sua destinação é importante verificar as suas características para optar pela melhor escolha, pois segundo a NDWCH (1998) quando se pensa em plano de gerenciamento de resíduos sólidos é necessário caracterizar a forma, a quantidade e a qualidade dos resíduos para assim determinar as possíveis formas de disposição tornando-se, portanto, imprescindível a caracterização do material, sendo as análises de microscopia eletrônica de varredura e raios-X importantes ferramentas na caracterização dos RETAs.

As características químicas como concentração de metais pesados, $\mathrm{pH}$, demanda bioquímica de oxigênio (DBO), demanda química de oxigênio (DQO), nitrogênio e fósforo são parâmetros que afetam mais as opções de disposição e reuso do que os processos de manuseio, adensamento e desidratação (SCALIZE, 2003). As características físicas, tais como a resistência específica, a taxa de sedimentação e a concentração de sólidos são determinantes na seleção e dosagem ótima dos condicionantes utilizados como auxiliares dos processos de adensamento e desaguamento, na determinação do potencial de desaguamento e na estimativa da quantidade de torta gerada, enquanto características como viscosidade, densidade, tamanho e distribuição das partículas são importantes na seleção do processo utilizado para desaguamento (CORNWELL et al., 1987).

Dentre as operações unitárias de tratamento de água a maior quantidade de resíduos é gerada no decantador, em termos mássicos, e nos filtros, em termos volumétricos. Devido a essas características optou-se, nesse trabalho, pela coleta dos resíduos dos decantadores. Tais resíduos são formados principalmente por compostos orgânicos provenientes da fonte de abastecimento e por resíduos dos produtos químicos utilizados no tratamento, portanto, dentre os vários fatores que influenciam as características do RETA inclui-se a qualidade da água bruta e o tipo de produto químico utilizado no tratamento, podendo haver variações de acordo com a mudança desses fatores.

Sabendo que ao caracterizar um material torna-se possível determinar as melhores formas de disposição ou reutilização dele, o presente trabalho teve por objetivo caracterizar o resíduo oriundo do decantador da ETA Engenheiro Rodolfo José da Costa, que utiliza como coagulante sulfato de alumínio, com o intuito de utiliza-lo no tratamento do efluente de um sistema de lagoas de estabilização. 


\section{MATERIAIS E MÉTODOS:}

Os resíduos foram obtidos na ETA Eng. Rodolfo José da Costa (ETA Meia Ponte), que capta água do rio Meia Ponte, está localizada em Goiânia, Goiás, Brasil, com capacidade de $2 \mathrm{~m}^{3} / \mathrm{s}$, abastece cerca de $50 \%$ da população goianiense.

O total de água produzida por essa ETA, anualmente, está em torno de $70.000 .000 \mathrm{~m}^{3}$. Segundo Souza (2011), a perda de água referente às descargas dos decantadores, limpeza dos filtros, limpeza dos tanques de produtos químicos e manutenções da ETA representa em média 2,8\%.

A ETA Meia Ponte é classificada como ciclo completo, sendo composta por calha Parshall, com medição ultrassônica da vazão, floculadores hidráulicos, com 4 (quatro) câmaras cada um, 3 decantadores convencionais, com raspadores mecanizados (velocidade constante de aproximadamente $1,0 \mathrm{~m} / \mathrm{min}$ ) e descargas de fundo semi-contínuas realizadas a cada 1 hora independentemente da posição da ponte raspadora e da qualidade da água bruta. O sistema de filtração é composto por filtros com 2 células e dupla camada de filtração (areia e antracito).

A estação possui ainda 2 lagoas de secagem de lodo, para onde são destinados os resíduos provenientes da descarga dos decantadores e da lavagem de filtros. O funcionamento das lagoas é alternado, uma lagoa recebe os resíduos enquanto na outra ocorre a secagem do resíduo anteriormente enviado a ela. Ao secar, o resíduo é retirado da lagoa e disposto ao seu lado e, a lagoa em que se procedeu a limpeza passa a receber os resíduos enquanto na outra é iniciado o processo de secagem. Essa alternância ocorre a cada ano, aproximadamente.

O coagulante utilizado na ETA é o sulfato de alumínio, e a coagulação ocorre pelo mecanismo de varredura, não tendo sido utilizado durante o período da pesquisa nenhum auxiliar de floculação, bem como carvão ativado ou outro produto no tratamento da água antes da realização da desinfecção. O clima da região onde se localiza a ETA é do tipo, segundo Köppen, quente e semi-úmido com estação seca bem definida, de maio a setembro, e regime pluviométrico tropical, com temperatura média anual de $23,2^{\circ} \mathrm{C}$, com as médias mínimas e máximas de $17,9^{\circ} \mathrm{C}$ e $29,8^{\circ} \mathrm{C}$, respectivamente (BRASIL, 1992).

O resíduo foi coletado durante a descarga do decantador, sendo que nesse processo o lodo é descartado através de uma descarga de fundo em uma galeria que o encaminha para a entrada da lagoa de lodo. O RETA foi coletado na galeria de drenagem durante a descarga de fundo.

Foram realizadas 8 coletas simples entre os meses de maio/12 a maio/13 para as análises do lodo in natura, já para a análise do resíduo seco foram realizadas duas coletas, com intervalo de uma semana entre elas, em agosto/12 e em cada coleta foram obtidos resíduos provenientes da

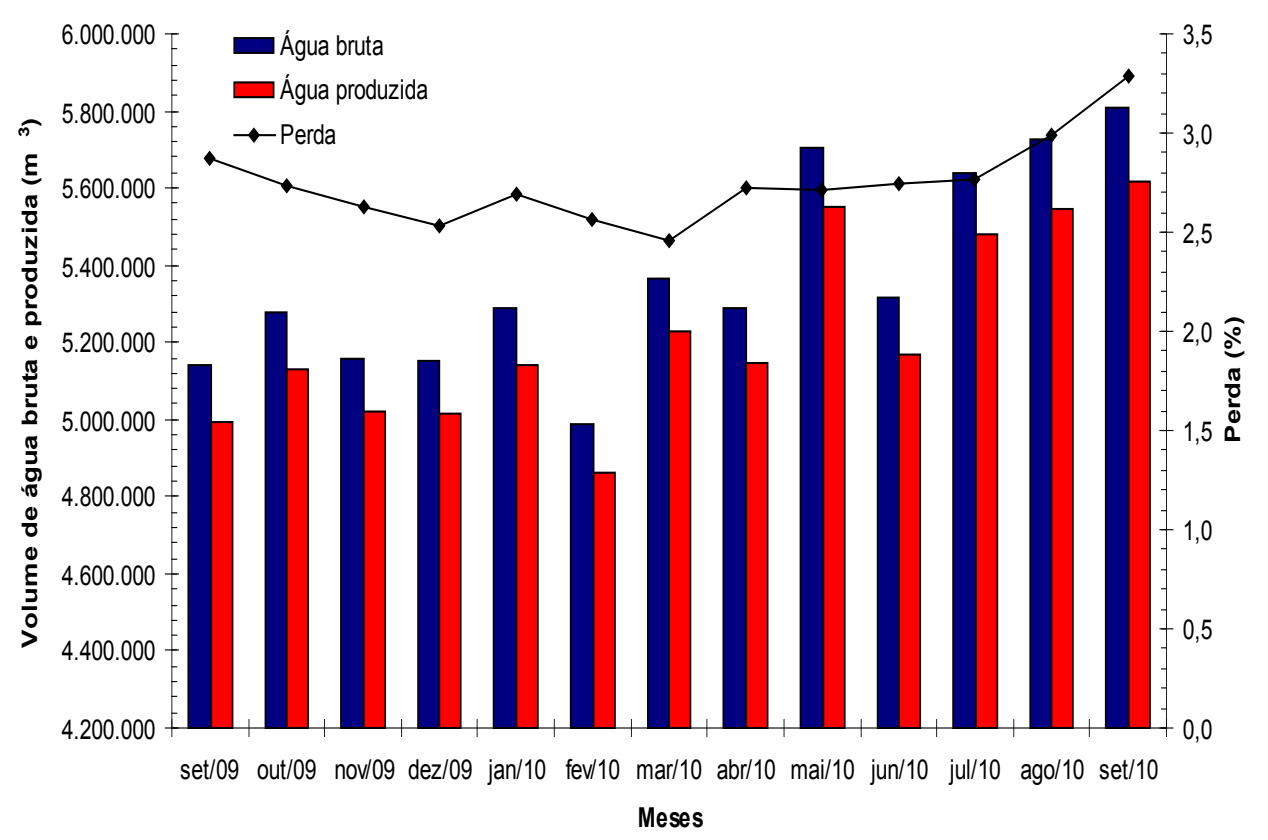

Figura 1 - Volume de água bruta e de água produzida pela ETA - Meia Ponte e a porcentagem da água bruta perdida. 
Tabela 1. Métodos utilizados na determinação dos parâmetros físico-químicos do RETA.

\begin{tabular}{ll}
\hline Parâmetros & $\mathrm{N}^{\circ}$ do método (APHA, 2005) \\
\hline DBO & $5210 \mathrm{~B}$ \\
DQO & $5220 \mathrm{D}$ \\
Fósforo total & $4500 \mathrm{P.B} \mathrm{e} \mathrm{P.E}$ \\
Nitrato & $4500 \mathrm{NO3} \mathrm{B}$ \\
Nitrogênio amoniacal orgânico & $4500 \mathrm{Norg} \mathrm{B}$ \\
pH & 4500 \\
Sólidos Totais & $2540 \mathrm{~B}$ \\
Sólidos Fixos Totais & $2540 \mathrm{E}$ \\
Sólidos Voláteis Totais & $2540 \mathrm{E}$ \\
Sólidos Suspensos Totais & $2540 \mathrm{D}$ \\
Sólidos Suspensos Fixos & $2540 \mathrm{E}$ \\
Sólidos Suspensos Voláteis & $2540 \mathrm{E}$ \\
\hline
\end{tabular}

Tabela 2. Análises realizadas no RETA após secagem em estufa à $70^{\circ} \mathrm{C}$.

\begin{tabular}{|c|c|}
\hline Análise & Equipamento ou Norma \\
\hline $\begin{array}{l}\text { Distribuição } \\
\text { granulométrica }\end{array}$ & $\begin{array}{l}\text { Analisador, difração a laser Coulter, registro do sinal de difração pelo } \\
\text { software LS200, "Variablespeedfluid module plus" }\end{array}$ \\
\hline Massa específica & Norma NBR 6508 de 1984 \\
\hline Limite de plasticidade & Norma NBR 7180 de 1984 \\
\hline $\begin{array}{l}\text { Morfologia e } \\
\text { microestrutura }\end{array}$ & Microscópio eletrônico de varredura, marca Rontech - Alemanha \\
\hline $\begin{array}{l}\text { Composição e } \\
\text { distribuição relativa } \\
\text { dos elementos }\end{array}$ & $\begin{array}{l}\text { Microscópio eletrônico de varredura combinado a raio-x de energia } \\
\text { dispersiva, marca Rontech - Alemanha }\end{array}$ \\
\hline $\begin{array}{l}\text { Análise mineralógica } \\
\text { qualitativa }\end{array}$ & Equipamento de Raio-x, marca Rigaku - EUA \\
\hline
\end{tabular}

descarga dos 3 decantadores. O resíduo in natura foi caracterizado de acordo com os parâmetros da Tabela 1 e as análises realizadas no Laboratório de Saneamento da Escola de Engenharia Civil UFG, conforme APHA (2005). Parte do resíduo foi seco a $70^{\circ} \mathrm{C}$ e submetido as análises que constam na Tabela 2, e realizadas nos laboratórios da Universidade da Beira Interior (Covilhã-Portugal)

\section{RESULTADOS E DISCUSSÃO}

A caracterização química do RETA "in natura", após ser preparado para concentração de SST de aproximadamente 3,0 g.L. - $^{-1}$ (Tabela 03) demonstrou a presença de nitrogênio e fósforo, esse último em maior concentração. A contribuição desses nutrientes na composição do RETA pode ser resultado da precipitação desses elementos contidos na água bruta, influenciada pelo característica da bacia de contribuição. O valor de DQO foi próximo ao encontrado por Scalize (2003) e Rosario (2007) quando analisaram o RETA de uma estação que também empregava o sulfato de alumínio como coagulante.

$\mathrm{O}$ pH do resíduo variou entre 6,57 e 7,66, faixa típica encontrada na literatura para esse tipo de resíduo. A principal preocupação com o $\mathrm{pH}$ advém da toxicidade do alumínio presente em sua composição, pois sabe-se que sua especiação é dependente do $\mathrm{pH}$, com maior presença de espécies solúveis em faixas de $\mathrm{pH}$ inferiores a 6,0.

Os sólidos são os materiais mais comuns em RETAs, eles advém principalmente da água 
Tabela 3. Caracterização físico-química do RETA com SST de aproximadamente 3,0 g/L.

\begin{tabular}{|c|c|c|c|c|c|}
\hline \multirow[t]{2}{*}{ Parâmetro } & \multicolumn{3}{|c|}{ Resultados obtidos } & \multirow{2}{*}{$\begin{array}{l}\text { Número de } \\
\text { amostras }\end{array}$} & \multirow{2}{*}{$\begin{array}{l}\text { Desvio } \\
\text { Padrão }\end{array}$} \\
\hline & Média & Mínimo & Máximo & & \\
\hline SST (g.L $\left.L^{-1}\right)$ & 2,94 & 2,69 & 3,2 & 8 & 0,24 \\
\hline Nitrogênio amoniacal (mg. $\left.\mathrm{L}^{-1}\right)$ & 0,56 & ND & 3,08 & 8 & 1,07 \\
\hline Nitrato $\left(m g . L^{-1}\right)$ & 0,36 & ND & 1,5 & 8 & 0,56 \\
\hline Fósforo total $\left(\mathrm{mg} . \mathrm{L}^{-1}\right)$ & 5,7 & 3,7 & 7,5 & 8 & 1,47 \\
\hline $\mathrm{pH}$ & 7,06 & 6,57 & 7,66 & 8 & 0,42 \\
\hline $\mathrm{DQO}\left(\mathrm{mg} . \mathrm{L}^{-1}\right)$ & 1100 & 1050 & 1132 & 4 & 35,57 \\
\hline
\end{tabular}

ND - não detectado

bruta, mas a adição de produtos químicos colabora para a quantidade de sólidos formada. A Tabela 4 apresenta os valores de sólidos obtidos em quatro coletas simples, sem nenhuma alteração no resíduo coletado, ou seja, nessas análises não foi modificada a concentração de SST, e a porcentagem de suas parcelas fixas e voláteis em relação a parcela total. Foi possível verificar maior concentração de sólidos fixos, representando cerca de $80 \%$ dos sólidos e que cerca de $20 \%$ era representada por sólidos voláteis, com exceção da amostra 1 , demonstrando que a maior parte da fração sólida é representada por substancias minerais. Esse resultado é compatível com o descrito por ASCE (1996), que diz que a maior parte dos sólidos contidos no RETA é inerte, de modo que a relação sólidos voláteis totais e sólidos totais é geralmente inferior a 30\%. Do resíduo estudado apenas a relação SV e ST do resíduo 01 apresentou-se em 32,7\%.

$\mathrm{Na}$ análise de sólidos suspensos também se verificou a maior parcela de sólidos suspensos fixos (acima de $80 \%$ ), que incluem resíduos inorgânicos (silte, areia, argila e hidróxidos de metais) e menos de $20 \%$ de SSV, representado a matéria orgânica (matéria coloidal floculada e compostos que contribuem para cor). Os resultados condizem com os valores encontrados por Souza (2011) ao analisar o resíduo da mesma ETA.
Os sólidos são os materiais mais comuns em RETAs, eles advém principalmente da água bruta, mas a adição de produtos químicos colabora para a quantidade de sólidos formada. A Tabela 4 apresenta os valores de sólidos obtidos em quatro coletas simples, sem nenhuma alteração no resíduo coletado, ou seja, nessas análises não foi modificada a concentração de SST, e a porcentagem de suas parcelas fixas e voláteis em relação a parcela total. Foi possível verificar maior concentração de sólidos fixos, representando cerca de $80 \%$ dos sólidos e que cerca de $20 \%$ era representada por sólidos voláteis, com exceção da amostra 1, demonstrando que a maior parte da fração sólida é representada por substancias minerais. Esse resultado é compatível com o descrito por ASCE (1996), que diz que a maior parte dos sólidos contidos no RETA é inerte, de modo que a relação sólidos voláteis totais e sólidos totais é geralmente inferior a 30\%. Do resíduo estudado apenas a relação SV e ST do resíduo 01 apresentou-se em 32,7\%.

$\mathrm{Na}$ análise de sólidos suspensos também se verificou a maior parcela de sólidos suspensos fixos (acima de 80\%), que incluem resíduos inorgânicos (silte, areia, argila e hidróxidos de metais) e menos de $20 \%$ de SSV, representado a matéria orgânica (matéria coloidal floculada e compostos que contribuem para cor). Os resultados condizem

Tabela 4. Caracterização de sólidos nas amostras do RETA in natura, ou seja, sem alteração na concentração de SST.

\begin{tabular}{lcccccccc}
\hline \multirow{2}{*}{ Parâmetro } & \multicolumn{9}{c}{ Resíduo 01 } & \multicolumn{3}{c}{ Concentração } \\
\cline { 2 - 9 } & \multicolumn{9}{c}{$\left(\mathrm{mg} \cdot \mathrm{L}^{-1}\right)$} & $(\%)$ & $\left(\mathrm{mg} \cdot \mathrm{L}^{-1}\right)$ & $(\%)$ & $\left(\mathrm{mg} . \mathrm{L}^{-1}\right)$ & $(\%)$ & $\left(\mathrm{mg} . \mathrm{L}^{-1}\right)$ & $(\%)$ \\
\hline ST & 9083 & 100 & 2882 & 100 & 2230 & 100 & 3726 & 100 \\
SFT & 6117 & 67,3 & 2624 & 91,0 & 1940 & 86,9 & 2984 & 80,1 \\
SVT & 2966 & 32,7 & 258 & 9,0 & 290 & 13,1 & 742 & 19,9 \\
SST & 5050 & 100 & 2120 & 100 & 2700 & 100 & 1670 & 100 \\
SSF & 4160 & 82,3 & 1710 & 80,6 & 2220 & 82,0 & 1400 & 83,8 \\
SSV & 900 & 17,7 & 410 & 19,4 & 490 & 18,2 & 270 & 16,2 \\
\hline
\end{tabular}


com os valores encontrados por Souza (2011) ao analisar o resíduo da mesma ETA.

A análise granulométrica do resíduo demonstrou uma larga distribuição de tamanho de partículas, que pode ser melhor visualizada pelas imagens do MEV na figura 5, estando compreendidas na faixa de $1,91 \mu \mathrm{m}$ a $409,6 \mu \mathrm{m}$, com cerca de $1 \%$ abaixo de $2 \mu \mathrm{m}$, sendo classificadas como argilas pela NBR 6502 (1995). Pela mesma classificação o conteúdo de silte está em aproximadamente $39 \%$ (valores entre 2 e $60 \mu \mathrm{m}$ ) e a maior parcela, $60 \%$, é representada por areia (valores entre 60 e $2000 \mu \mathrm{m})$. Ippolito; Barbarick e Elliot (2011) ao levantar os valores encontrados por uma série de pesquisadores também verificaram que a maior quantidade de resíduo é representada por areia (69\%), seguida de silte (17\%) e argila (14\%), porém a quantidade encontrada nesta pesquisa (1\%) foi menor que a encontrada por eles (14\%).

No ensaio de limite de plasticidade o RETA teve comportamento não plástico, diferindo dos resultados encontrados por Oliveira, Machado e Holanda (2004) e Teixeira et. al (2011) na caracterização de resíduos, obtidos a partir do tratamento com sulfato de alumínio, para uso na indústria cerâmica, porém o resíduo obtido nos dois estudos apresentaram alta concentração de partículas inferiores a $2 \mu \mathrm{m}$, diferente do que foi encontrado no resíduo estudado nesta pesquisa, apesar de, assim como o RETA das pesquisas referenciadas, não ter tido nenhum pré-tratamento. $\mathrm{O}$ termo plasticidade está relacionado à propriedade de uma massa argilosa mudar a sua forma sem ruptura, sob a ação de uma força externa, bem como a retenção da nova forma após retirada da força aplicada. Essa característica é importante na aplicação em indústrias cerâmicas, porém considerando o uso do resíduo no tratamento de águas residuais pode não ter significância. Já com relação a massa específica o valor obtido para o resíduo neste estudo, $2,54 \mathrm{~g} . \mathrm{cm}^{-3}$, apresentou valor próximo ao encontrado por Oliveira, Machado e Holanda (2004), 2,63 g. $\mathrm{cm}^{-3}$, e por Titshall e Hughes (2005), que ao avaliar o resíduo de ETA na África obteve valores entre 2,02 a 2,43 g. $\mathrm{cm}^{-3}$

A distribuição dos elementos na superfície do RETA demonstrou que o resíduo é composto pelos elementos químicos $\mathrm{O}(67,53 \%), \mathrm{Al}(13,22 \%)$, $\mathrm{Si}(10,06 \%), \mathrm{Fe}(8,01 \%), \mathrm{K}(0,40 \%), \mathrm{Ca}(0,38 \%)$ e $\mathrm{Ti}(0,40 \%)$. Wang et. al (2013) também verificaram no RETA os elementos O (41,86\%), Fe $(26,56 \%)$, $\mathrm{Al}(9,06 \%)$, Si $(4,76 \%)$ e Ca $(1,79 \%)$, porém com maior presença de ferro que de alumínio devido ao RETA analisado ter sido obtido de uma estação que operava tanto com coagulante a base de ferro quanto de alumínio. Outros autores verificaram a presença dos elementos $\mathrm{Al}, \mathrm{Fe}$ e $\mathrm{Si}$, estando presentes na forma de hidróxidos, sendo suas quantidades dependentes do metal usado no coagulante (OLIVEIRA; MACHADO; HOLANDA, 2004; BABATUNDE et. al, 2009; TEIXEIRA et. al, 2011; IPPOLITO; BARBARICK; ELLIOT, 2011).

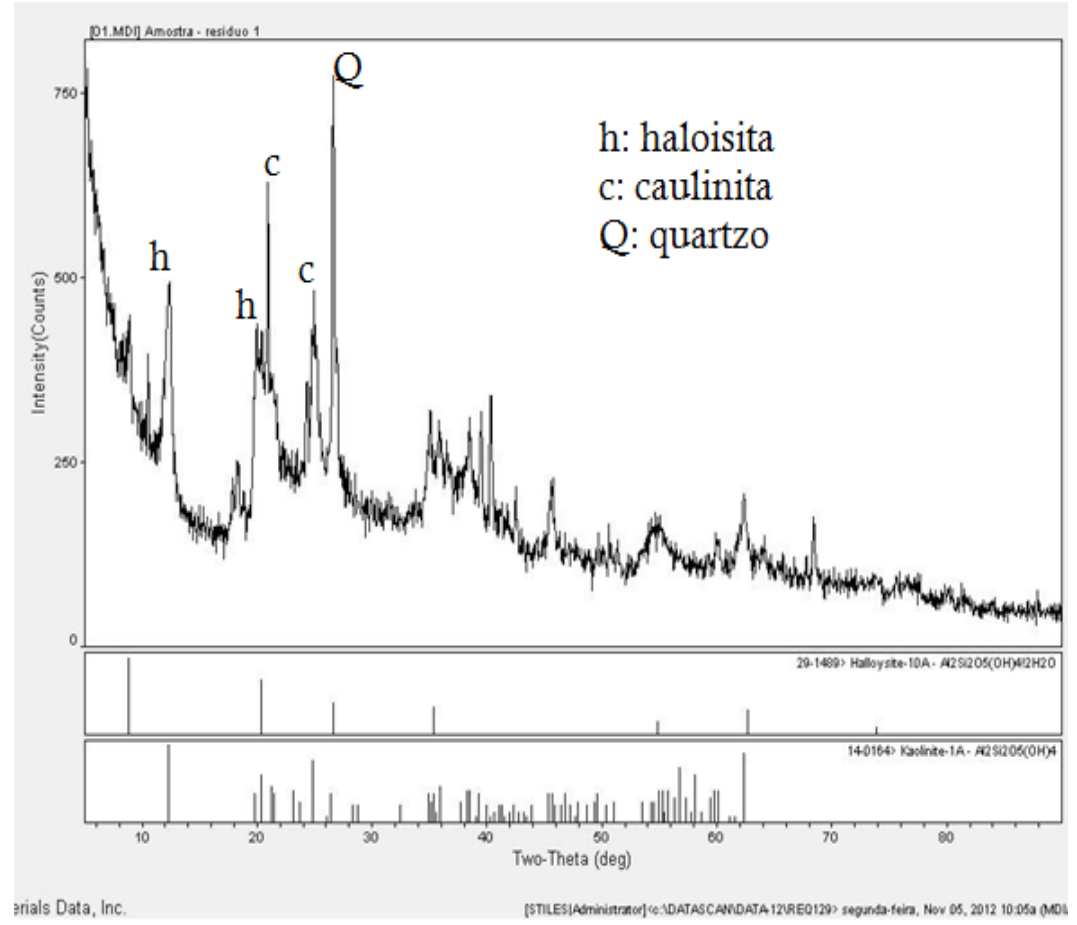

Figura 2. Difractograma de raios-X do RETA, obtido após secagem do resíduo à $70^{\circ} \mathrm{C}$. 


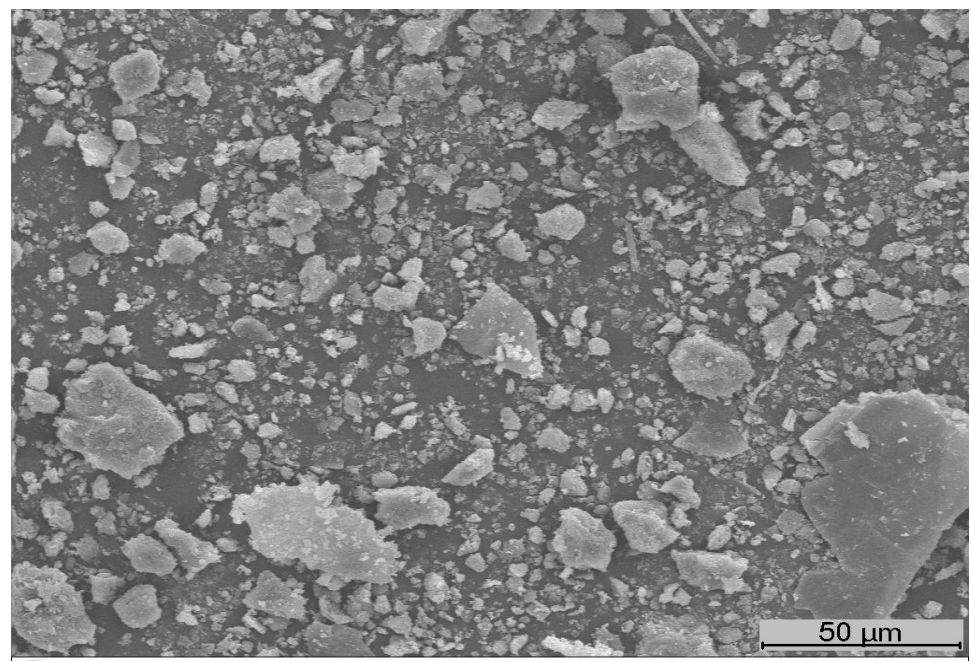

Figura 3. Imagem de Microscopia Eletrônica de Varredura do RETA, seco a $70^{\circ} \mathrm{C}$, com magnificação de 500x, permitindo visão geral das partículas que compõem o RETA.

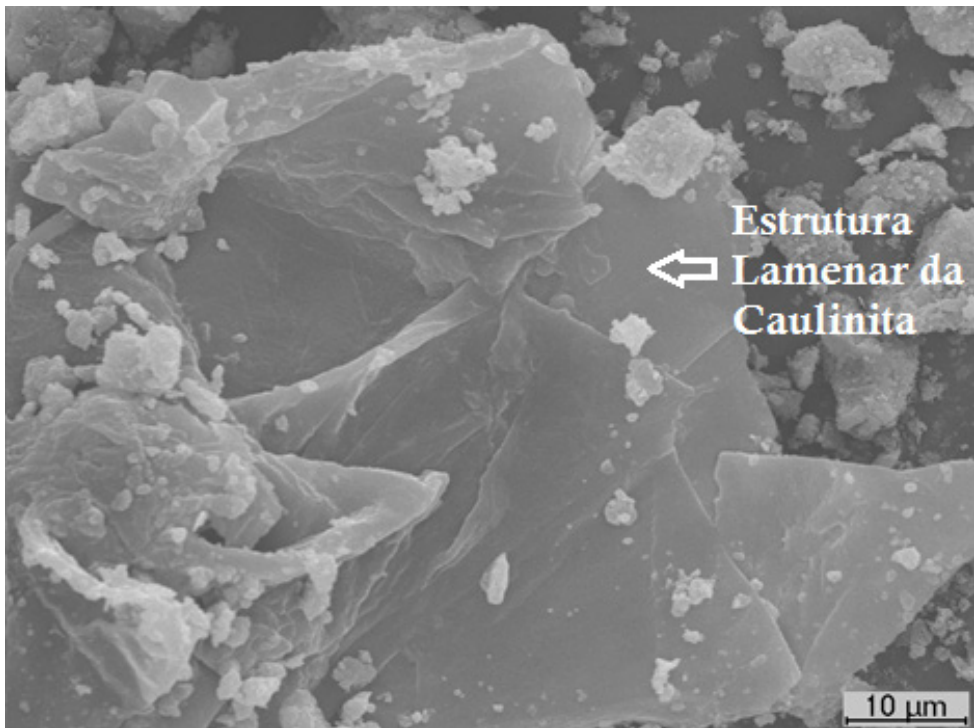

Figura 4. Imagem de Microscopia Eletrônica de Varredura do RETA, seco a $70^{\circ} \mathrm{C}$, com magnificação de $1500 \mathrm{x}$, permitindo visualizar a caulinita que compõe a estrutura do RETA.

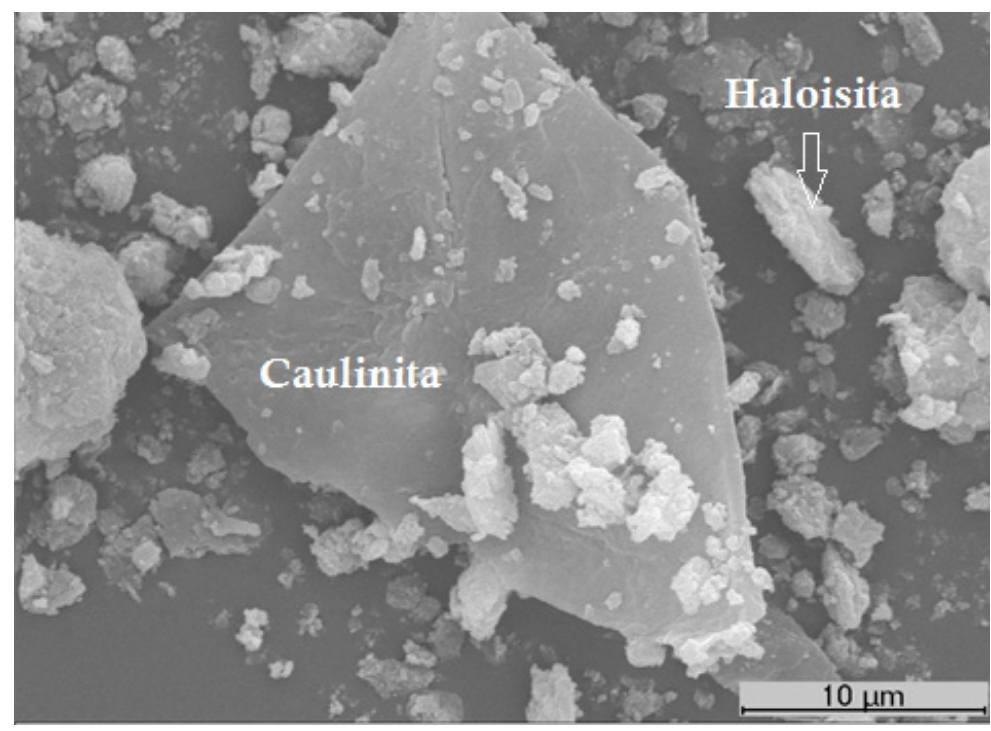

Figura 5. Imagem de Microscopia Eletrônica de Varredura do RETA, seco a 70 C, com magnificação de 3000x, permitindo visualizar a caulinita e a haloisita que compõe a estrutura do RETA. 
O alumínio é conhecido por desempenhar importante função na adsorção/precipitação de fósforo, assim materiais ricos em alumínio são capazes de promover sua remoção, a depender do $\mathrm{pH}$ da solução com favorecimento para baixos valores de $\mathrm{pH}$, podendo ser aplicados no tratamento de águas residuais, que apresentam, normalmente, altas concentrações de fósforo, sendo essa capacidade de adsorção não afetada pela adsorção dos íons cloreto, nitrato, sulfato e por substâncias orgânicas (URANO; TACHIKAWA, 1991). Com relação a presença do elemento fósforo na composição do RETA, apesar de detectado na análise físicoquímica não foi percebida sua contribuição na análise de Espectroscopia de energia dispersiva de raios-X (EDS), situação diferente da reportada por Oliveira, Machado e Holanda (2004).

Na Figura 2 é possível verificar, pelo difractograma de raios-X, estrutura amorfa com picos referentes aos minerais caulinita e haloisita, também é possível identificar a presença de quartzo. Porém, a caulinita é o mineral predominante na amostra assim como foi encontrado nos trabalhos de Oliveira, Machado e Holanda (2004) e Teixeira et. al (2011). Segundo Ippolito, Barbarick e Elliott (2011) devido a sua natureza amorfa e a presença dos hidróxidos de ferro e alumínio, os RETAs tem alta capacidade de adsorver aníons, o que torna interessante o seu uso no tratamento de águas residuais.

A caulinita é um silicato lamelar (filossilicato) que apresenta estrutura formada por uma camada de silicato simples e uma camada de $\mathrm{\gamma}$-gibsita $\left(\mathrm{Al}(\mathrm{OH})_{3}\right)$. A composição química teórica da caulinita é de $39,50 \%$ de $\mathrm{Al}_{2} \mathrm{O}_{3}, 46,54 \%$ de $\mathrm{SiO}_{2}$ e $13,96 \%$ de $\mathrm{H}_{2} \mathrm{O}$, no entanto podem ser verificadas pequenas variações em sua composição (LUZ e LINS, 2005). O grupo funcional mais reativo na caulinita é a hidroxila, capaz de participar em muitas reações químicas, bem como na troca iônica (LEE, 1999). Segundo estudos realizados por Chen; Butler e Stumm (1973) a remoção de fósforo pelo mineral caulinita ocorre pelo processo de adsorção, ou ao menos, pela formação de camadas superficiais amorfas. Já a haloisita é uma variedade polimorfa da caulinita, podendo estar associada a ela, sendo possível sua identificação apenas com o uso de imagens do $\mathrm{MEV}$, pois a haloisita apresenta-se na forma tubular e pode ser desidratada facilmente, enquanto a caulinita apresenta-se na forma de partículas hexagonais (LUZ; LINS, 2005).

Pelas imagens no MEV foi possível verificar partículas desuniformes e com larga distribuição granulométrica (Figura 3), reforçando os resultados obtidos na análise de granulometria e tendo característica semelhante ao verificado no trabalho de Zhao et. al (2006), que também utilizou MEV na análise um resíduo de ETA, que emprega sais de alumínio como coagulante, verificando que o resíduo era virtualmente amorfo, com forma e tamanho variável. Também foi possível verificar que o RETA em estudo é formado pelos minerais caulinita e haloisita, Figuras 4 e 5, sugerindo o seu potencial uso na remoção de fósforo pelo processo de adsorção.

\section{CONCLUSÕES}

O resíduo avaliado neste estudo apresentou características similares aos demais RETAs encontrados na literatura, com a exceção de ser não plástico, porém essa característica é mais importante quando se avalia a sua reutilização na indústria cerâmica, podendo ser indiferente para sua aplicação no tratamento de esgoto.

A composição granulométrica demonstrou partículas de dimensões variadas, com presença de argila (1\%), silte (39\%) e areia (60\%), sendo majoritária a presença de areia. A caulinita mostrou ser o mineral predominante, o que viabiliza o uso do resíduo na remoção de fósforo pelo processo de adsorção. Essa capacidade de remoção do nutriente fósforo também pôde ser verificada por meio das análises de composição química em que foram detectados os elementos O, Al, Si, Fe, K, Ca e Ti, tendo grande importância a presença de alumínio, pois materiais ricos nesse elemento são capazes de agregar o elemento fósforo, com a vantagem de que a adsorção de outros íons, principalmente íons nitrato e sulfato, não prejudicam a remoção de fósforo. Pela analise de raios-X também foi possível verificar a estrutura amorfa do material, sendo a hidroxila o grupo funcional mais reativo, capaz de participar em muitas reações químicas, bem como na troca iônica.

Pelas análises realizadas é possível perceber que o RETA pode ser aplicado no tratamento de águas residuais, pois suas características permitem a remoção de fósforo, que é um elemento muito importante quando se trata da eutrofização de corpos receptores, e de outros elementos presentes nas águas residuais, como o nitrogênio que também colabora para o processo de eutrofização, auxiliando no aumento da eficiência de tratamento das ETEs. 


\section{REFERÊNCIAS}

AMERICAN SOCIETY OF CIVIL ENGINEERS ASCE; AMERICAN WATER WORKS ASSOCIATION - AWWA. Technology Transfer Handbook : Management of Water Treatment Plant Residuals. New York, 1996.

APHA. STANDARD METHODS FOR THE EXAMINATION OF WATER AND WASTEWATER, 21 ed., 2005.

\section{ASSOCIAÇÃO BRASILEIRA DE NORMAS TÉC-} NICAS. NBR 6502, 1995.

ASSOCIAÇÃO BRASILEIRA DE NORMAS TÉCNICAS. NBR 10004: Resíduos Sólidos - Classificação. Rio de Janeiro, 2004.

BABATUNDE, A.O.; ZHAO, Y.Q, BURKE, A.M.; MORRIS, M.A.; HANRAHAN, J.P. Characterization of aluminium-based water treatment residual for potential phosphorus removal in engineered wetlands. Environmental Pollution, v. 157, p. 2830-2836, 2009.

BRASIL. Ministério da Agricultura e Reforma Agrária. Secretaria Nacional de Irrigação. Departamento Nacional de Meteorologia. Normais climatológicas: 1961-1990. Brasília, DF, 1992.

CHEN, Y. S. R.; BUTLER, J. N.; STUMM, W. Adsorption of phosphate on Alumina and Kaolinite from Dilute Aqueous Solution. Journal of Colloid and Interface Science, v. 43, n. 2, p. 421 - 436, 1973.

CORNEWELL, D.A.; BURMASTER, J.W.; FRANCIS, J.L.; FRIEDLINE, J.C.; HOUCK, C.; KING, P. H.; KNOCKE, W.R.; NOVAK, J.T.; ROLAN, A.T.; SAN GIACOMO, R. Committee Report: Research Needs for Alum Sludge Discharge. Journal AWWA, v. 79, p. $99-105,1987$.

EPA. Drinking Water Treatment Plant Residuals Management Technical Report, 2011.

IPPOLITO, J.A.; BARBARICK, K. A.; ELLIOT, H. A. Drinking Water Treatment Residuals: A Review of Recent Uses. Journal Environmental Quality, v. 40, p. 1-12, 2011.

LEE, J.D. Química Inorgânica não tão concisa. São Paulo: Edgard Blücher, 1999.
LUZ, A.B.; LINS, F.A.D. Rochas e Minerais Industriais: usos e especificações. Rio de Janeiro: CETEM/MCT, 2005.

NDWCH - National Drinking Water Clearinghouse. Tech Brief: Water Treatment Plant Residuals Management, n. 7, p. 1-4, mar. 1998.

OLIVEIRA, E.M.S.; MACHADO, S.Q.; HOLANDA, J.N.F. Caracterização de resíduo (lodo) proveniente de estação de tratamento de águas visando sua utilização em cerâmica vermelha. Cerâmica, v. 50, p. 324-330, 2004.

ROSÁRIO, C.G.A. Avaliação da Disposição de Lodo Gerado numa Estação de Tratamento de Água em Reator Anaeróbio de Fluxo Ascendente e Manto de Lodo (UASB). São Paulo, SP, 2007. Originalmente apresentada como dissertação de mestrado, Programa de Pós-Graduação em Engenharia Hidráulica e Saneamento, USP, 2007.

SCALIZE, P.S. Disposição de Resíduos Gerados em Estações de Tratamento de Água em Estações de Tratamento de Esgoto. São Carlos, SP, 2003. Originalmente apresentada como tese de doutorado, Escola de Engenharia de São Carlos, Universidade de São Paulo, 2003.

SILVA JUNIOR, A.P. Tratabilidade do lodo de decantadores convencional e de alta taxa. Originalmente apresentada como dissertação de mestrado, Faculdade de Engenharia Civil, Universidade Estadual de Campinas, Campinas, 2003

SOUZA, L.M. Estudo da aplicação do resíduo de decantador de ETA como auxiliar de floculação no tratamento de água. Originalmente apresentado como dissertação de mestrado, Programa de Pós - Graduação em Engenharia do Meio Ambiente, Universidade Federal de Goiás, 2011.

TEIXEIRA, S.R.;. SANTOS, G.T. A; SOUZA, A.E.; ALESSIO, P.; SOUZA, S.A.; SOUZA, N.R. The effect of incorporation of a Brazilian water treatment plant sludg eon the properties of ceramic materials. Applied Clay Science, v. 53, p. 561-565, 2011.

TITSHALL L.W.; HUGHES J.C. Characterisation of some South African water treatment residues and implications for land application. Water SA, n.3, 299-308, 2005. 
URANO, K.; TACHIKAWA, H. Process development for removal and recovery of phosphorus from wastewater by a new adsorbent. 2. Adsorption rates and break through curves. Industrial Engineering Chemistry Research, v. 30, p. 1897 - 1899, 1991.

ZHAO, Y.Q; YANG, Y; TOMLINSON, D; KENNEDY, S. Dewatered alum sludge: a potential adsorbent for phosphorus removal. Water Science \&Technology, v. 52, n. 10-11, p 525-532, 2006.

WANG, C.; GAO, S.; PEI, Y.; ZHAO, Y. Use of drinking water treatment residuals to control the internal phosphorus loading from lake sediments: Laboratory scale investigation. Chemical Engineering Journal, v. 225, p. 93-99, 2013. 